\title{
DIFERENTES FORMULAÇÕES DE PROGRAMAÇÃO LINEAR INTEIRA PARA A ROTEIRIZAÇÃO NO TRANSPORTE DE FUNCIONÁRIOS
}

\author{
Tarek Nasser Sati (GTAO/UFPR) tarek.sati@ufpr.br \\ Cassius Tadeu Scarpin (GTAO/UFPR) cassiusts@ufpr.br \\ José Eduardo Pécora Junior (GTAO/UFPR) pecora@ufpr.br \\ Roza Maria Zoellner Lopes (GTAO/UFPR) roza.lopes@ufpr.br
}

\section{Resumo}

Diversas empresas oferecem opção de transporte para seus funcionários. Por ser uma tarefa realizada diariamente, otimizar este processo pode resultar em uma considerável diminuição de custos para a empresa. Neste artigo é comparada a roteirização do transporte de funcionários, em uma empresa na cidade de Curitiba, por meio de diferentes modelos exatos de Programação Linear Inteira mista (PLIM). São realizadas três diferentes propostas de resolução para o caso, utilizando metodologias baseadas na formulação dos seguintes problemas: Travelling Salesman Problem (TSP) ou Problema do Caixeiro Viajante; Vehicle Routing Problem (VRP) ou Problema de Roteirização de Veículos; e P-median Problem (PMP) ou Problema das P-medianas. Os resultados mostraram grande diferença no tempo computacional quando se divide o problema em duas fases, porém relativamente pequena diferença na distância percorrida. Todos os testes computacionais foram processados no software Gurobi, utilizando o Software Excel e o suplemento Open Solver como interface para entrada de dados.

Palavras-Chaves: Problema de Roteirização no Transporte Escolar, Problema do Caixeiro Viajante, Roteirização de Veículos, Programação Linear Inteira, Pesquisa Operacional.

\section{Introdução}

Em Pesquisa Operacional, uma das áreas mais estudadas é a de roteirização no transporte, onde existe grande variabilidade em relação aos tipos de problema e diferentes métodos de resolução para cada um deles. Neste artigo são analisados apenas modelos exatos de Programação Linear Inteira Mista (PLIM) com o objetivo de solucionar um problema de roteirização de veículos.

Os dados utilizados provêm de uma empresa fictícia, que busca os funcionários em suas casas com três diferentes veículos. O objetivo principal é comparar os resultados obtidos quando o problema é formulado em uma fase (agrupamento e roteirização dos funcionários decididas de 
forma simultânea no modelo) e em duas fases (primeiro faz-se o agrupamento de funcionários e, depois, com os resultados, para cada agrupamento, faz-se a roteirização de coleta dos funcionários). Para resolver o caso desta empresa, foram propostas três diferentes formulações, onde foram utilizados os modelos exatos do caixeiro viajante (TSP); roteamento de veículos (VRP); e P-medianas (PMP). O caso desta empresa é conhecido como problema de roteirização no transporte escolar, que de acordo com Bektas (2006), consiste em um problema de múltiplos caixeiros viajantes (mTSP) e difere-se do TSP pela utilização de múltiplos veículos.

O TSP foi proposto por Dantzig, Fulkerson e Johnson (1954) e tem como objetivo encontrar a menor rota possível. Aborda-se o problema onde um vendedor ambulante parte de uma cidade, passa por todas as cidades presentes no problema (uma única vez) e então retorna à inicial.

Segundo Laporte (2009), o VRP é uma generalização do TSP, com grau de dificuldade aumentado. Devido à esta complexidade, no geral, são utilizados algoritmos com heurísticas para a resolução. O primeiro artigo publicado sobre o problema de roteirização de veículos foi realizado por Dantzig e Ramser (1959), que teve como resultado a otimização da rota de caminhões que realizavam entregas de um terminal para estações de serviço. Segundo Bektas (2006), o mTSP difere do VRP nas restrições de capacidade, que são removidas, porém as formulações propostas pelo VRP também são válidas para o caso do mTSP.

Apesar de definido quinze anos antes, com Hakimi (1964) e Hakimi (1965), a primeira vez que o problema das P-medianas foi formulado como um modelo de programação linear inteiro foi por ReVelle e Swain (1970). O problema consiste em um modelo de localização de instalações, onde são formados grupos entre estas, de forma a minimizar suas distâncias entre as instalações e suas respectivas medianas. Cada instalação pode ser uma possível mediana e o número total de medianas é definido por $\mathrm{P}$.

Tiburcio (2012) realizou o roteamento do problema do transporte para o caso de uma empresa em Curitiba, porém utilizou em sua formulação heurísticas de aproximação, deixando um GAP para a utilização de modelos exatos. Na revisão literária, utilizando a base de dados Web Of Science não foi encontrado nenhum tipo de aplicação de roteirização para buscar funcionários na cidade de Curitiba, portanto um artigo comparando formulações exatas em um software de uso comum é considerado de grande valia. 


\section{O problema}

O problema consiste em realizar a roteirização de coleta de funcionários de uma empresa na cidade de Curitiba. Esta empresa busca os funcionários em suas casas e para isso conta com três vans com capacidade para sete pessoas cada. O objetivo principal é minimizar a distância total percorrida pelas vans, porém, também deve ser analisado o tempo de viagem de cada um dos funcionários, tendo em vista que um longo tempo de espera não é vantajoso.

A abordagem proposta foi construída de maneira estratégica, visando possibilitar a comparação dos resultados de maneira visual, utilizando do Google Maps. A escolha do número de funcionários para a roteirização foi definida de maneira que possibilitasse a solução com tempo computacional aceitável. A distribuição dos funcionários foi definida, de forma fíctícia devido ao sigilo de dados, com pontos no mapa que forçam os veículos a percorrer grande parte da cidade de maneira relativamente uniforme, similar ao problema real. O número de veículos também foi definido de acordo com os existentes na empresa, no caso, são utilizadas vans.

A localização da empresa e a distribuição de seus funcionários pela cidade de Curitiba é apresentada na Figura 1, onde a empresa é identificada por um marcador distinto.

Figura 1 - Mapa geográfico dos funcionários e da empresa

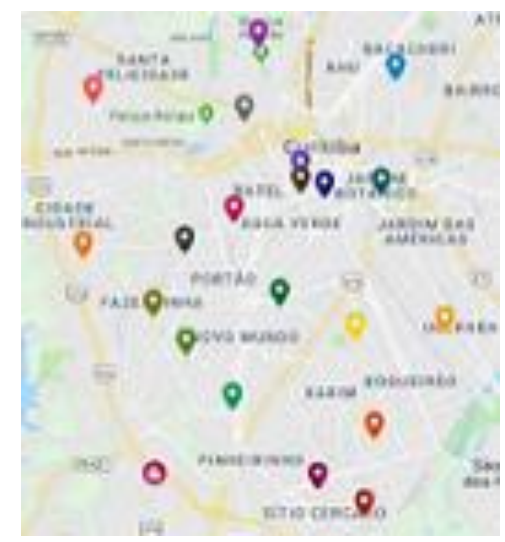

Fonte: O autor (2020)

O objetivo principal deste artigo é comparar a resolução deste problema por meio de três diferentes propostas, duas delas para solucionar o problema com todas as suas características e outra apenas para efeito comparativo. Todas foram abordadas utilizando apenas modelos exatos de programação linear. São elas:

- Um modelo do caixeiro viajante (TSP), sem agrupamento e sem capacidade. Usa-se esta abordagem apenas para mensurar o aumento da distância percorrida pelo uso de 
mais veículos, tendo em vista que este modelo resulta na melhor (menor) rota possível.

- Da decomposição do problema em duas fases. A primeira consiste em agrupar os funcionários em três diferentes grupos de no máximo sete pessoas, cada um dos grupos é designado a uma determinada van. O modelo utilizado nesta fase é o baseado no Problema das P-medianas. A segunda fase consiste na roteirização de cada um dos clusters encontrados na primeira fase, o modelo utilizado foi o do caixeiro viajante (TSP). Cada um dos agrupamentos consiste em um problema diferente.

- Por meio da otimização buscada diretamente do modelo de roteirização de veículos (VRP) com restrição de capacidade dos veículos, considerando três veículos com capacidade de sete pessoas cada.

\section{Metodologia}

Nesta seção apresenta-se a proposta de abordagem para o problema de roteirização no transporte de funcionários. O capítulo está dividido em duas subseções, primeiramente é feita uma classificação metodológica da pesquisa, seguida pelos modelos e métodos utilizados para resolução.

\subsection{Classificação metodológica}

A pesquisa presente neste trabalho é de natureza aplicada, ou seja, possui aplicação prática, focada em resolver problemas reais. Por se tratar de um problema matemático, e o fato de que, segundo Lakatos e Marconi (2003), problemas matemáticos são dedutivos, que partem de premissas verdadeiras e estabelecem relações lógicas entre estas, este trabalho possui um processo de raciocínio de dedução.

É realizada com uma abordagem quantitativa, constituída de hipóteses claramente especificadas e variáveis operacionalmente definidas, que por meio de uma medição objetiva e quantificação dos resultados, atinge boa precisão. Isso faz com que esta abordagem evite distorções na etapa de análise e interpretação de dados, garantindo margem de segurança em relação às inferências (GODOY, 1995). 
O trabalho tem o objetivo classificado como pesquisa exploratória, para Gil (2002), o objetivo principal deste tipo de pesquisa é o aprimoramento de ideias, a fim de tornar o tema mais explícito e constituir hipóteses, sendo bastante flexíveis.

A classificação quanto aos procedimentos técnicos é de um estudo de caso, que de acordo com Gil (2002), é um estudo exaustivo de poucos objetos, permitindo um conhecimento amplo e detalhado, sendo considerado útil para formular hipóteses e desenvolver teorias.

\subsection{Modelos propostos}

Como descrito na seção anterior, foram utilizadas três diferentes formulações, o modelo matemático realizado em cada uma delas é descrito nesta subseção, respectivamente à ordem citada na seção anterior. O objetivo com esta roteirização é fazer com que o veículo de transporte passe por todos os pontos (funcionários) apenas uma vez, tendo como ponto inicial e final a empresa, sem a ocorrência de subciclos. A função objetivo minimiza a distância total percorrida e as restrições garantem que as necessidades operacionais do problema sejam respeitadas. Para os modelos propostos os parâmetros utilizados são os seguintes:

- $\quad d_{i j} \quad$ Distância do nó $i$ para o nó $j$;

- $n \quad$ Número de nós visitados (funcionários + empresa);

- $\quad p \quad$ Número de medianas;

- $\quad m \quad$ Número de veículos.

E as variáveis de decisão são:

$$
\begin{aligned}
& -x_{i j}=\left\{\begin{array}{l}
1, \text { se o arco do nó } i \text { para o nó } j \text { é utilizado } \\
0, \text { caso contrário }
\end{array}\right. \\
& -x_{i j k}=\left\{\begin{array}{l}
1, \text { se o arco do nó } i \text { para o nó } j \text { utilizando o veículo } k \text { é utilizado } \\
0, \text { caso contrário }
\end{array}\right. \\
& -u_{i} \text { e } u_{j} \quad \text { Variáveis Dummy de chegada e saída ao nós } i \text { e } j, \text { respectivamente. }
\end{aligned}
$$


Para calcular as distâncias entre cada ponto, foram utilizadas as coordenadas de graus decimais (DD), tendo como resultado as distâncias euclidianas. Segundo Tiburcio (2012), para a cidade de Curitiba estas distâncias correspondem a $65 \%$ da distância real, percentual este que foi agregado às distâncias calculadas na função objetivo. O modelo matemático utilizado na primeira formulação está descrito abaixo, adaptado do modelo proposto por Miller, Tucker e Zemlin (1960).

$\operatorname{Min} \sum_{i=1}^{n} \sum_{j=1}^{n} d_{i j} x_{i j} / 0,65$

Sujeito a:

$\sum_{j=1}^{n} x_{i j}=1, i=1, \ldots, n$

$\sum_{i=1}^{n} x_{i j}=1, j=1, \ldots, n$

$u_{i}-u_{j}+n x_{i j} \leq n-1, i, j=2, \ldots, n$

$x_{i j} \in\{0,1\}$

As restrições (1) e (2) garantem que o veículo chegue e saia apenas uma vez de cada local visitado; a restrição (3) é conhecida como restrição de MTZ e garante que não haverá subciclos. A variável $x_{i j}$ é binária.

$\mathrm{Na}$ primeira fase da segunda formulação, dividida em duas fases, as distâncias não são divididas por 0,65 , pois o resultado da função objetivo não possui relevância para o problema. Esta fase serve apenas para agrupar os funcionários, grupos estes que na segunda fase compõem cada um dos veículos. O modelo desta fase provém do modelo das P-medianas proposto por ReVelle e Swain (1970) e está descrito abaixo.

$\operatorname{Min} \sum_{i=1}^{n} \sum_{j=1}^{n} d_{i j} x_{i j}$

Sujeito a:

$\sum_{i=1}^{n} x_{i j}=1, j=1, \ldots, n$ 
$\sum_{i=1}^{n} x_{i i}=p$

$\sum_{i=1}^{n} x_{i j} \leq 7 j=1, \ldots, n$

$x_{i j} \leq x_{i i} \quad i, j=1, \ldots, n$

$x_{i j} \in\{0,1\}$

A restrição (1) garante que cada funcionário seja designado apenas à uma mediana; a restrição (2) define o número de medianas; a restrição (3) restringe o número máximo de funcionários por mediana (capacidade do veículo); a restrição (4) garante que um funcionário só possa ser designados a outro, caso este segundo seja uma mediana. A variável $x_{i j}$ é binária e o valor de

P é igual a três (três veículos).

A segunda fase desta formulação é similar à primeira formulação, a diferença é que, ao invés de roteirizar todos os funcionários, são realizadas três diferentes roteirizações, cada uma entre a empresa e um dos grupos encontrados na primeira fase.

A terceira formulação possibilita solucionar o problema da empresa em apenas uma fase. $\mathrm{O}$ modelo utilizado provém do proposto por Christofides e Eilon (1969), com a adaptação de se utilizar a restrição de subciclos de MTZ. O modelo da terceira formulação é descrito abaixo.

$\operatorname{Min} \sum_{i=1}^{n} \sum_{j=1}^{n} \sum_{k=1}^{m} d_{i j} x_{i j k} / 0,65$

Sujeito a:

$$
\begin{aligned}
& \sum_{j=2}^{n} x_{1 j k}=1, k=1, \ldots, m \\
& \sum_{i=2}^{n} x_{i 1 k}=1, k=1, \ldots, m
\end{aligned}
$$


$\sum_{j=1}^{n} \sum_{k=1}^{m} x_{i j k}=1, \quad i=2, \ldots, n$

$\sum_{i=1}^{n} \sum_{k=1}^{m} x_{i j k}=1, j=2, \ldots, n$

$\sum_{i=1}^{n} x_{i r k}=\sum_{j=1}^{n} x_{r j k}, r=2, \ldots, n, k=1, \ldots, m$

$u_{j}+(n-m) \cdot \sum_{k=1}^{n} x_{i j k} \leq n-m-1, i, j=2, \ldots, n, \quad \mathrm{i} \neq j$

$\sum_{i=1}^{n} \sum_{j=1}^{n} x_{i j k} \leq 7, k=1, \ldots, m$

$x_{i j k} \in\{0,1\}$

As restrições (1) e (2) garantem que para cada veículo, haja somente uma chegada e uma partida da empresa; as restrições (3) e (4) garantem que cada funcionário seja buscado uma única vez, independente para qual veículo seja; a restrição (5) garante que o veículo que chegue em uma determinada residência de um funcionário seja o mesmo que saia; a restrição (6) restringe subciclos (MILLER, TUCKER E ZEMLIN, 1960); a restrição (7) limita a capacidade do veículo à sete funcionários. A varável $x_{i j k}$ é binária.

\section{Resultados e discussões}

Após apresentado o problema e os modelos utilizados, nesta seção são apresentados e analisados os resultados após a implementação das três diferentes formulações. Para resolver cada uma das formulações, foi utilizado o Software Excel como interface de dados, com o suplemento OpenSolver (opensolver.org), e o software de solução do modelo PLIM foi o Gurobi. Para a realização dos testes foi utilizado um computador com processador Intel Core i7 com CPU de 2.20GHz e memória RAM de 8GB. 
Primeiramente analisa-se o resultado do TSP para apenas um veículo, que teve como a distância total percorrida $63,4 \mathrm{~km}$. Este resultado consiste na menor distância possível passando por todos os pontos, porém não é visto como uma possível resposta para o caso desta empresa, apenas como valor de referência para comparação. A rota percorrida pelo veículo, neste resultado, é apresentada na Figura 2.

Figura 2 - Roteirização utilizando o TSP

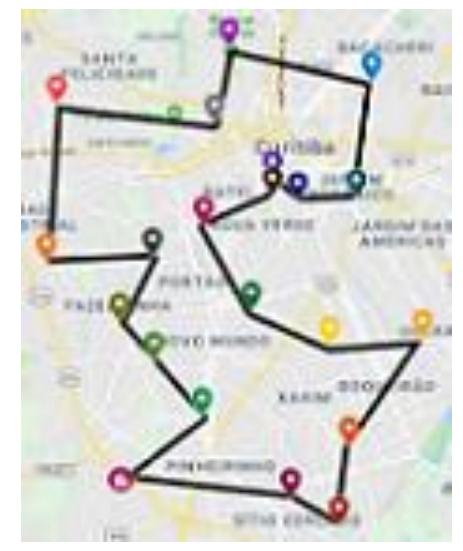

Fonte: O autor (2020)

A segunda abordagem, como um dos dois possíveis resultados para o problema, traz o benefício é de uma resposta muito rápida, menos de 10 segundos. Em contrapartida, por dividir o problema em duas fases, há perda de qualidade em sua resposta, o resultado obtido é mostrado na Figura 3, onde a área hachurada corresponde ao agrupamento da primeira fase e consequentemente, a área atendida por cada veículo. O veículo 1 (verde) percorre $25 \mathrm{~km}$, transportando 6 funcionários, o veículo 2 (roxo) percorre $37,1 \mathrm{~km}$, transportando 7 funcionários, e o veículo 3 (laranja) percorre 31,9km, transportando 7 funcionários. A soma das distâncias percorridas por cada veículo é de $94 \mathrm{~km}$.

Figura 3 - Roteirização em duas fases, utilizando o PMP e o TSP

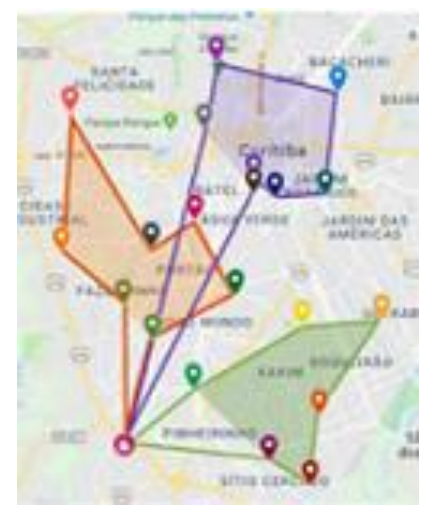

Fonte: O autor (2020) 
$\mathrm{Na}$ terceira formulação, e segundo resultado possível para o problema, temos como resposta a melhor solução possível para três veículos com capacidade de sete pessoas. A desvantagem deste modelo é que o tempo de obtenção de resposta é de 8 horas a mais em relação à segunda formulação. Entretanto a distância total percorrida pelos 3 veículos é de 90,2km, sendo 40,8km com 7 funcionários no veículo 1 (roxo), 25km e 6 funcionários no veículo 2 (azul) e 24,4km e 7 funcionários no veículo 3 (verde). A Figura 4 apresenta a resposta obtida.

\section{Figura 4 - Roteirização utilizando o VRP}

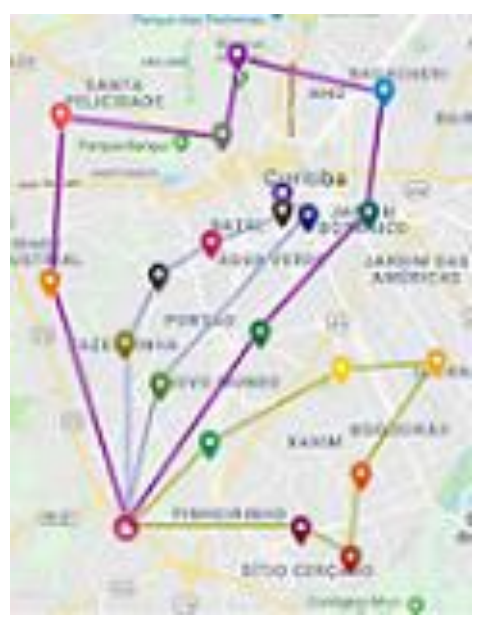

Fonte: O autor (2020)

Comparando os dois possíveis resultados, notamos que a diferença da distância percorrida é pequena, a segunda formulação teve um total percorrido de $94 \mathrm{~km}$, enquanto a terceira, o total foi de $90,2 \mathrm{~km}$, cerca de $4 \%$ menor. A utilização de um modelo ou outro dependerá do tempo disponível, além do tamanho do problema a ser solucionado. Para o caso de uma empresa que realiza este roteamento com pouca frequência, e com um número de funcionários inferior a 20, é valido o uso do VRP com múltiplos veículos capacitados, porém, caso a roteirização seja mais frequente ou o quadro de funcionários superior, a solução pelo VRP torna-se inviável, tendo a resolução indicada pela divisão do problema em duas fases, agrupar e roteirizar.

\section{Conclusões}

Neste artigo foram utilizados três diferentes modelos matemáticos, onde foram observados os benefícios de cada um deles. O modelo do caixeiro viajante foi utilizado apenas como parâmetro de rota mínima possível, este modelo obteve uma resposta cerca de $30 \%$ melhor (menor distância total percorrida) que o VRP, porém, como consequência os funcionários 
estariam por um longo tempo dentro do veículo, além de ser necessário um veículo de maior capacidade. A decomposição do problema em duas fases resultou em uma rota com distância total percorrida $4 \%$ maior que o VRP, entretanto o tempo computacional necessário foi muito menor, 10 segundos contra 8 horas do VRP. A escolha da utilização de cada uma dependerá do tamanho do problema a ser resolvido e da disposição de tempo computacional para obtenção da solução.

\section{REFERÊNCIAS}

BEKTAS, Tolga. The multiple traveling salesman problem: An overview of formulations and solution procedures. Omega, v. 34, n. 3, p. 209-219, 2006.

CHRISTOFIDES, N., EILON, S. An Algorithm for the Vehicle-dispatching Problem. Journal of the Operational Research Society, v. 20, p. 309-318, 1969.

DANTZIG, G. B.; FULKERSON, D. R.; JOHNSON, S. M. Solution of a Large-Scale Traveling Salesman Problem. Operations Research, v. 2, p. 393-410, 1954.

DANTZIG, G. B.; RAMSER, J. H. The Truck Dispatching Problem. Management Science, v. 6, n. 1, p. $80-91,1959$.

GIL, Antonio Carlos. Como elaborar projetos de pesquisa. $4^{\text {a }}$ Edição. São Paulo: Atlas S.A, 2002.

GODOY, Arilda S. Introdução à pesquisa qualitativa e suas possibilidades. Revista de Administração de Empresas, São Paulo, 1995, v.35, n. 2, p. 57-63.

Hakimi, S.L. Optimum Location of Switching Centers and the Absolute Centers and Medians of a Graph. Operations Research, v.12, p. 450-459, 1964.

Hakimi, S.L. Optimum Distribution of Switching Centers and Some Graph-Related Theoretic Problems. Operations Research, v.13, p. 462-475, 1965.

LAKATOS, Eva Maria; MARCONI, Marina de Andrade. Fundamentos de metodologia científica. 5. ed. São Paulo: Atlas, 2003.

LAPORTE, Gilbert. Fifty years of vehicle routing. Transportation Science, v. 43, n. 4, p. 408-416, 2009.

Miller, C.E.; Tucker, A.W. \& Zemlin, R.A. Integer programming formulation of traveling salesman problems. Journal of Association for Computing Machinery, v. 7, p. 326-9, 1960.

ReVelle, C.S. \& Swain, R.W. Central facilities location. Geographical Analysis, v. 2, p. 3042, 1970. 
TIBURCIO, Dalvana Matilde. Técnicas da pesquisa operacional na abordagem do problema de roteamento no transporte de funcionários de empresas. 2012. $86 \mathrm{f}$.

Dissertação (Mestrado) - Curso de Engenharia de Produção, Universidade Federal do Paraná, Curitiba, 2012. 